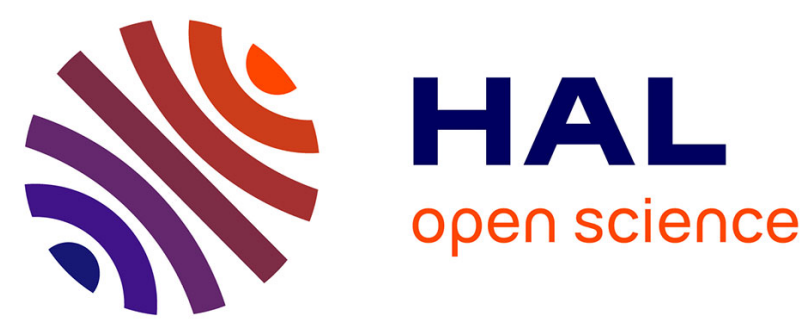

\title{
Turner à Sisteron
}

Roland Courtot

\section{To cite this version:}

Roland Courtot. Turner à Sisteron. Méditerranée: revue géographique des pays méditerranéens, 2004, Géosystèmes montagnards et méditerranéens. Un mélange offert à Maurice Jorda, 102 (1), pp.157 164. 10.3406/medit.2004.3351 . hal-01716308

\section{HAL Id: hal-01716308 \\ https://hal-amu.archives-ouvertes.fr/hal-01716308}

Submitted on 23 Feb 2018

HAL is a multi-disciplinary open access archive for the deposit and dissemination of scientific research documents, whether they are published or not. The documents may come from teaching and research institutions in France or abroad, or from public or private research centers.
L'archive ouverte pluridisciplinaire $\mathbf{H A L}$, est destinée au dépôt et à la diffusion de documents scientifiques de niveau recherche, publiés ou non, émanant des établissements d'enseignement et de recherche français ou étrangers, des laboratoires publics ou privés.

\section{(a)(1) $\$$}

Distributed under a Creative Commons Attribution - NonCommercial - NoDerivatives| 4.0 


\section{Turner à Sisteron.}

Courtot Roland. Turner à Sisteron. In: Méditerranée, tome 102, 1-2-2004. Geosystèmes montagnards et méditerranéens. Un mélange offert à Maurice Jorda. pp. 157-164 doi : 10.3406/medit.2004.3351

Autorisation accordée par l'éditeur

Roland Courtot, professeur émérite,

"TELEMMe, Aix Marseille Univ, CNRS, TELEMME, Aix-en-Provence, France"

\section{Introduction}

Les voyages en Europe continentale (France, Allemagne, Suisse, Italie) du peintre anglais William Turner on été une des sources de son inspiration pictural car il n'a cessé de remplir des carnets de dessins et d'aquarelles au cours de ses pérégrinations. Ces documents ont donné lieu à des études et à des expositions nombreuses, en particulier depuis la seconde moitié du $20^{\mathrm{e}}$ siècle. Il ne s'agit pas de revenir sur les analyses esthétiques de ces œuvres, largement développées par les historiens et les critiques d'art, mais de considérer l'approche géographique d'un site par le peintre, afin de comprendre comment il étudie et intègre la forme des objets paysagers dans sa transcription graphique et dans son appréhension de l'espace paysager. Alors qu'il a traversé plusieurs fois les Alpes française du nord pour se rendre en Suisse et en Italie (en 1802 et en 1836) et en a retiré un grand nombre d'œuvres (en particulier autour du massif du Mont Blanc; Hill, 2000) il a vu les Alpes du sud par deux fois : à l'occasion d'un voyage de Marseille à Gènes en 1828, pendant lequel il a beaucoup dessiné les paysages littoraux de la Côte d'azur et les Alpes maritimes, et en 1836 lors d'un voyage de retour, de Gènes à Grenoble, par Sisteron. C'est l'ensemble des croquis et aquarelles faits dans cette ville qui nous servira ici de corpus documentaire.

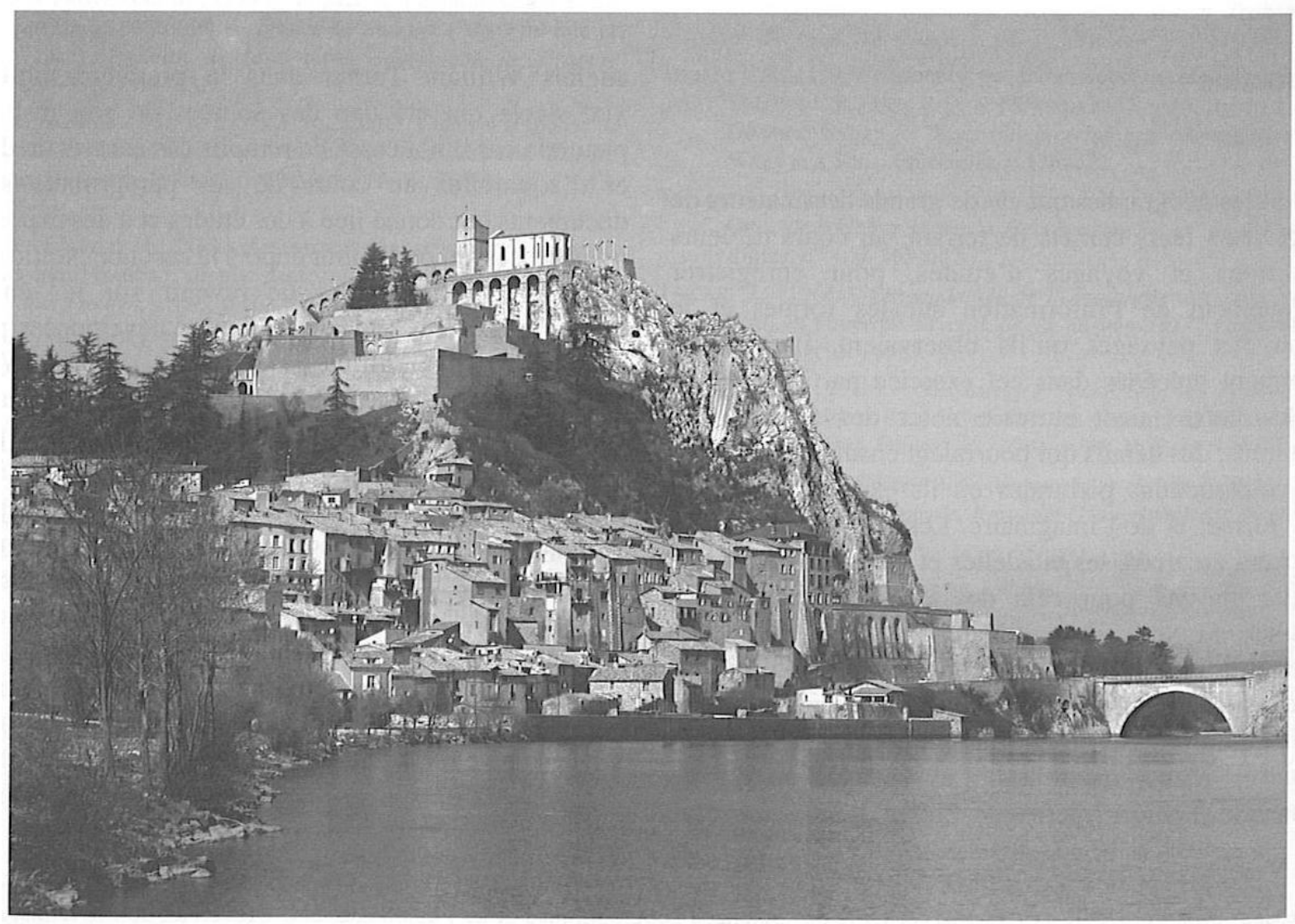

Fig 1 : Sisteron, la citadelle et la clue de la Durance vus du sud (photographie R. Courtot, mars 2004) 


\section{Les carnets de Turner et le site de Sisteron}

Notre source documentaire principale est donc le carnet CCXCV "From Genoa to Grenoble ", conservé à la Tate gallery de Londres, dans le fond Turner. Ce carnet a déjà été étudié par J. Guillebaud pour l'exposition qu'il a organisée sur “ Turner en France " à Paris en 1981 (Centre Culturel du Marais); il en a publié dans le catalogue de l'exposition un certain nombre de pages, ainsi que deux croquis noirs et blancs (tirés d'un autre carnet, CCCXLII) (fig.3 et 4) et deux aquarelles de Sisteron (Guillaud, 1981) (fig.5 et 6). En outre, ce carnet est, comme toutes les œuvres de Turner, directement consultable sur le site internet de la Tate gallery (tate.uk.org), mais la faible intensité du trait de crayon de Turner ne facilite pas toujours la lecture des dessins sur l'écran d'ordinateur. Le carnet fait 14,4cm sur 9,9 cm (c'est le format actuel de nos cartes postales et de nos photos papier), relié en parchemin, de Fratelli et Avondo (en filigrane), et porte une mention de M. Ruskin: "Generally careless, but grand at Sisteron" (généralement peu soigné, mais grand à Sisteron) Nous avons pu lever une hypothèque sur une partie non définie jusqu'ici du parcours de Turner entre la côte d'Azur et Sisteron : M. Guillebaud n'avait pu se prononcer pour l'itinéraire entre Cagnes sur mer et Mirabeau-Manosque (envisageant même l'hypothèse d'un passage par la route "Napoléon "). Nous avons maintenant la preuve qu'il est passé par Aix (deux pages du carnet en font foi de manière irréfutable), même si le parcours entre Cagnes et Aix n'est pas encore exactement identifié. D'Aix, il a donc suivi le val de Durance vers Sisteron, par la grande route, en dessinant au passage le château de Meyrargues, la clue de Mirabeau... Mais c'est le site de Sisteron qui, après la Côte d'Azur, a mérité le plus d'attention de sa part au cours de ce voyage.

C'est effectivement un site géographique notoire, qui sépare dans les Alpes du sud ce que les géographes ont appelé les pays de la moyenne Durance et les Préalpes méridionales sèches. La Durance traverse par une clue étroite (100m à la base) une barre rocheuse d'une centaine de mètres d'épaisseur de calcaire tithonique très résistant, orientée est-ouest et redressée à la verticale jusqu'à $600 \mathrm{~m}$ à l'ouest, et 829 à l'est. Il s'agit du flanc nord du synclinal du Jabron, axe le plus oriental du faisceau préalpin du Diois et des Baronnies. Au sens du relief plissé c'est un "crêt" dont les couches sont verticales et même un peu renversées, et la rivière le perce d'une " demi-cluse ", par " antécédence" ou " surimposition ". La ville est accrochée en rive droite de la Durance au pied sud de la barre occidentale, dominée par la citadelle ; en face, au delà du pont, le hameau de la Baume tire son nom du rocher qui le domine et dont les plans stratigraphiques verticaux ont été élargis en grottes par l'érosion. L'étroitesse de la clue, la dénivelée des barres rocheuses et de la citadelle (plus de $250 \mathrm{~m}$ presque à-pic), l'accumulation des fortifications, tout concourt à faire de Sisteron un site impressionnant (fig.1)

\section{Turner et la forme urbaine}

Pour étudier l'approche topographique du site, nous avons relevé à l'aide d'un plan ancien de Sisteron (tiré du cadastre de 1814), les points de vue successifs que Turner a adoptés pour dessiner la ville (cf. fig.2). En partant de l'hypothèse très plausible que le carnet a été rempli au fur et à mesure du cheminement de l'artiste, on obtient, en reportant ces points sur un plan, un véritable investissement de la ville, comme une armée venant mettre un siège, ou comme un photographe tournant autour de son sujet pour en capter tous les angles. Aucun dessin ne sera fait d'ailleurs à l'intérieur de la ville: le paysage urbain interne semble ne pas l'avoir intéressé, ce qui reste étonnant quant on sait le nombre de croquis " urbains" dont Turner a rempli ses carnets ; à croire qu'il n'est pas entré dans la ville, et qu'il n'a fait que “tourner autour”, alors qu'elle présente suffisamment de "monuments historiques" qui auraient pu retenir son attention (la cathédrale Notre Dame du Pommier, par exemple). C'est bien le site de la ville accrochée à son rocher et dominant sa clue qui retient toute son attention : 13 dessins de petit format figurent sur le carnet (15 même, si on tient compte de ce que 2 pages portent deux dessins) pris sur le vif, au fur et à mesure de l'approche et du cheminement. Cet intérêt manifesté pour le site de Sisteron (alors que dans ce même voyage la ville d'Aix-en-Provence n'a pratiquement pas retenu l'attention de l'artiste) 
s'explique par les qualités esthétiques du site, dont Turner sait qu'il pourra tirer des œuvres et des gravures. Cela vient aussi de ce qu'il arrive certainement à Sisteron " en connaissance de cause " : la ville et son site ont fait l'objet de plusieurs dessins de Richard Taylor en 1824, publiés dans un recueil à Londres, dont Turner a certainement eu connaissance (de Gombert, 1981, p.289). En tous cas, les points de vue adoptés par Taylor le sont aussi par Turner quelques années plus tard.

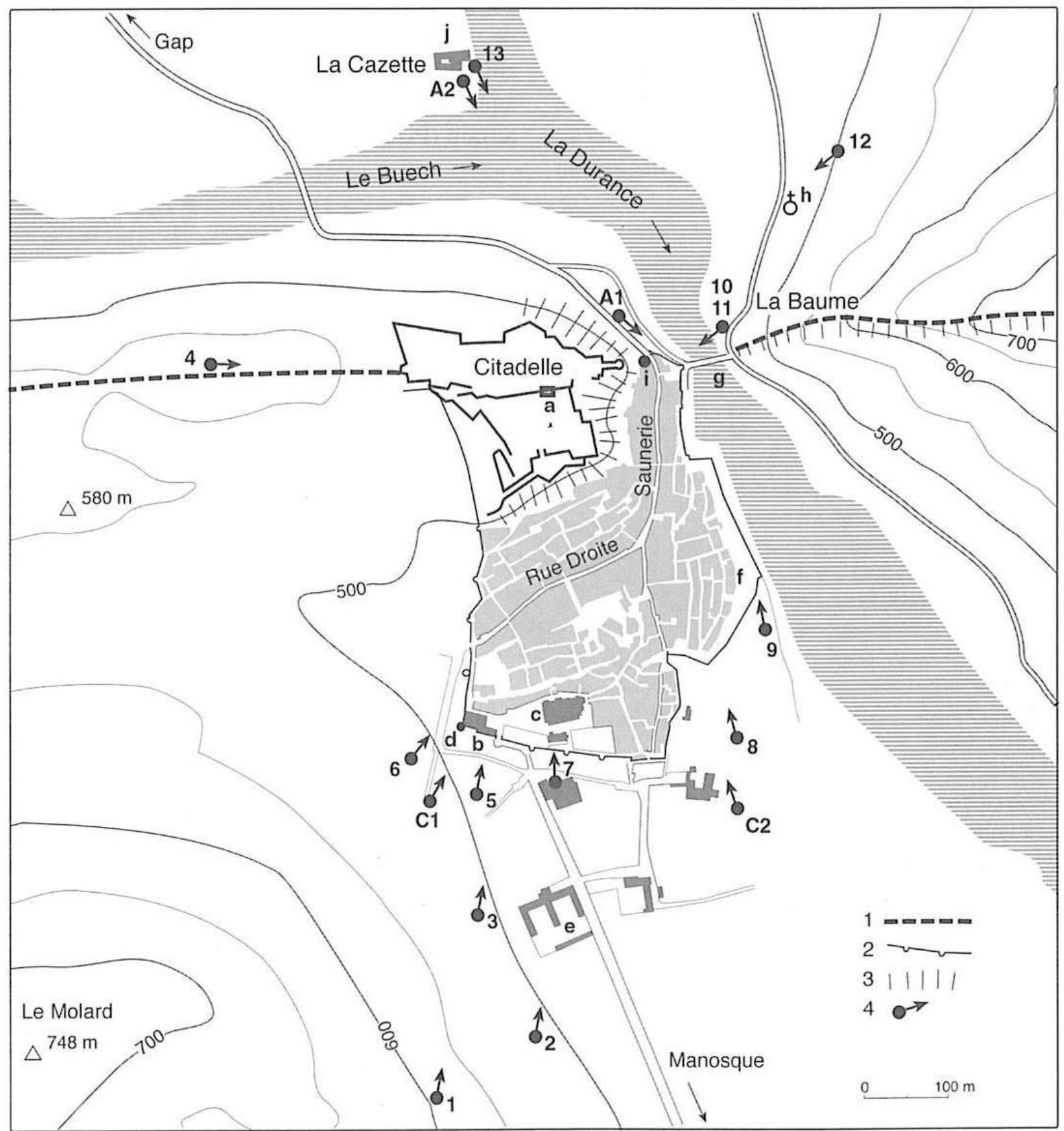

Fig. 2 : Les “points de vue " de Turner à Sisteron

Les numéros renvoient aux points de vue des dessins dans l'ordre des pages du carnet de croquis. A1 désigne l'Aquarelle depuis la Casette, A2 celle de la porte du Dauphiné.

Les éléments remarquables du site sur les croquis sont désigné par des lettres :

-a : donjon et église de la forteresse, -b : cathédrale, -c : prison, -d : tour des gendarmes, -e : hospice de la charité, -f : quartier des andrones, -g : pont sur la Durance, -h : couvent des dominicains, -i : tour du Dauphiné, -j : domaine et bastide de la Casette.

-1 : remparts et tours

-2 : escarpements rocheux

(Source : plan cadastral 1814 pour la ville, carte IGN 1/25000 ${ }^{\mathrm{e}}$ Sisteron 3339 OT 1997 pour les alentours) 
Turner dessine trois vues d'ensemble de loin (D29564 et D29562), lorsqu'il arrive par la route de Château-Arnoux : tout y est : la ville dans ses remparts, la citadelle dominante, le pont sur la Durance, le faubourg et le rocher de la Baume. Au premier plan à droite : l'hôpital de la charité, construit en 1720 à l'extérieur de la ville, est un bon repère. Puis trois vues rapprochées, en face de l'angle sud-ouest des remparts, gardé par la tour des gendarmes (dont le toit en forme de cône aplati est reconnaissable sur plusieurs dessins). Cela lui permet de mettre en scène à la fois les deux enfilades de tours de l'enceinte (vers la Durance et vers la citadelle) et quelques éléments majeurs de la topographie urbaine : la porte de Provence (ou "grand portail" de la ville), l'ancien hôtelDieu intra-muros et l'église cathédrale, Notre-Dame du Pommier. Aucun obstacle ne gène ces vues rapprochées, car Turner se trouve alors sur le "pré de foire", vaste espace dégagé au pied des remparts (rendu inconstructible par les règlements militaires) et situé à proximité de la sortie principale de la ville, lieu idéal pour les foires et les marchés.

Apparemment, entre deux de ces vues, il escalade la pente qui le domine au nord pour aller se jucher sur la crête à l'ouest de la citadelle : de là, une vue à niveau (66) lui montre le château de profil et lui permet de dessiner au nord, en perspective plongeante, le pont et la confluence du Buech, le rocher de la Baume et le couvent des dominicains (en rive gauche de la Durance).

Il redescend donc et continue à tourner autour de la ville en allant vers la rivière, du bord de laquelle il crayonne vers le nord les hautes maisons qui surplombent la Font chaude (le quartier aux "andrones", ruelles étroites et en partie couvertes, qui dévalent la pente urbaine) et le pont de la Baume (70 et 71). Il franchit le pont de la Baume d'où il croque rapidement la ville de face et sa sortie nord gardée par la porte St Etienne, et l'autre profil de la citadelle, en contre-plongée cette fois. Il poursuit son chemin en rive gauche vers le nord, pour dépasser le couvent des dominicains, et c'est là qu'il saisit le site de la clue vu de l'amont, en deux croquis (578 et 579), ou sont mis en évidence le rempart nord de la citadelle, la porte St Etienne, le pont de la Baume et le petit clocher fin des dominicains.

\section{Les développements du travail de terrain.}

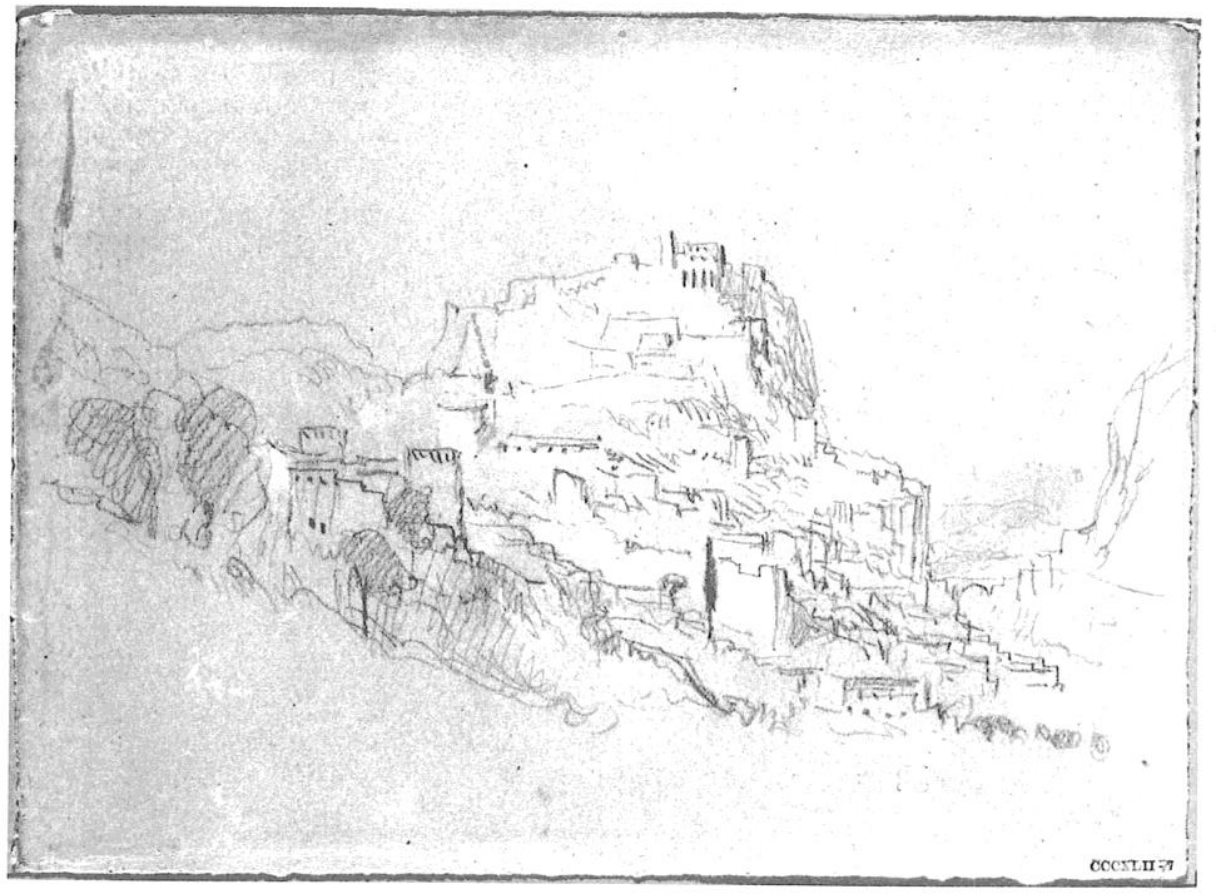

Fig.3 : Sisteron, dessin de Turner (carnet CCCXLII-7) (Crayon et craie blanche, 23 x $32 \mathrm{~cm}$ ), Tate gallery. Town, with Buildings on Mountain c.1830-41 D34191, Turner Bequest CCCXLII 7, CC-BY-NC-ND 3.0 http://www.tate.org.uk/art/artworks/turner-town-with-buildings-on-mountain-d34191 
Les études de la perspective sud de la ville ont été complétées par deux dessins au crayon rehaussé de blanc, de format plus important et plus " achevés " (Dessins du carnet CCCXLII-7 et 8, conservés à la Tate gallery) (Guillaud, 1981p.503 et504): soit qu'ils aient été commencés sur le terrain, soit qu'ils aient été construits plus tard en reprenant les croquis faits devant le rempart. La citadelle est dans les deux cas l'arrière plan central de la vue, mais l'axe de vue est dans un cas la tour de l'angle sud ouest de l'enceinte (porte de Provence), dans le second celle de l'angle sud est (porte Sauve) (cf. fig.3 et 4).
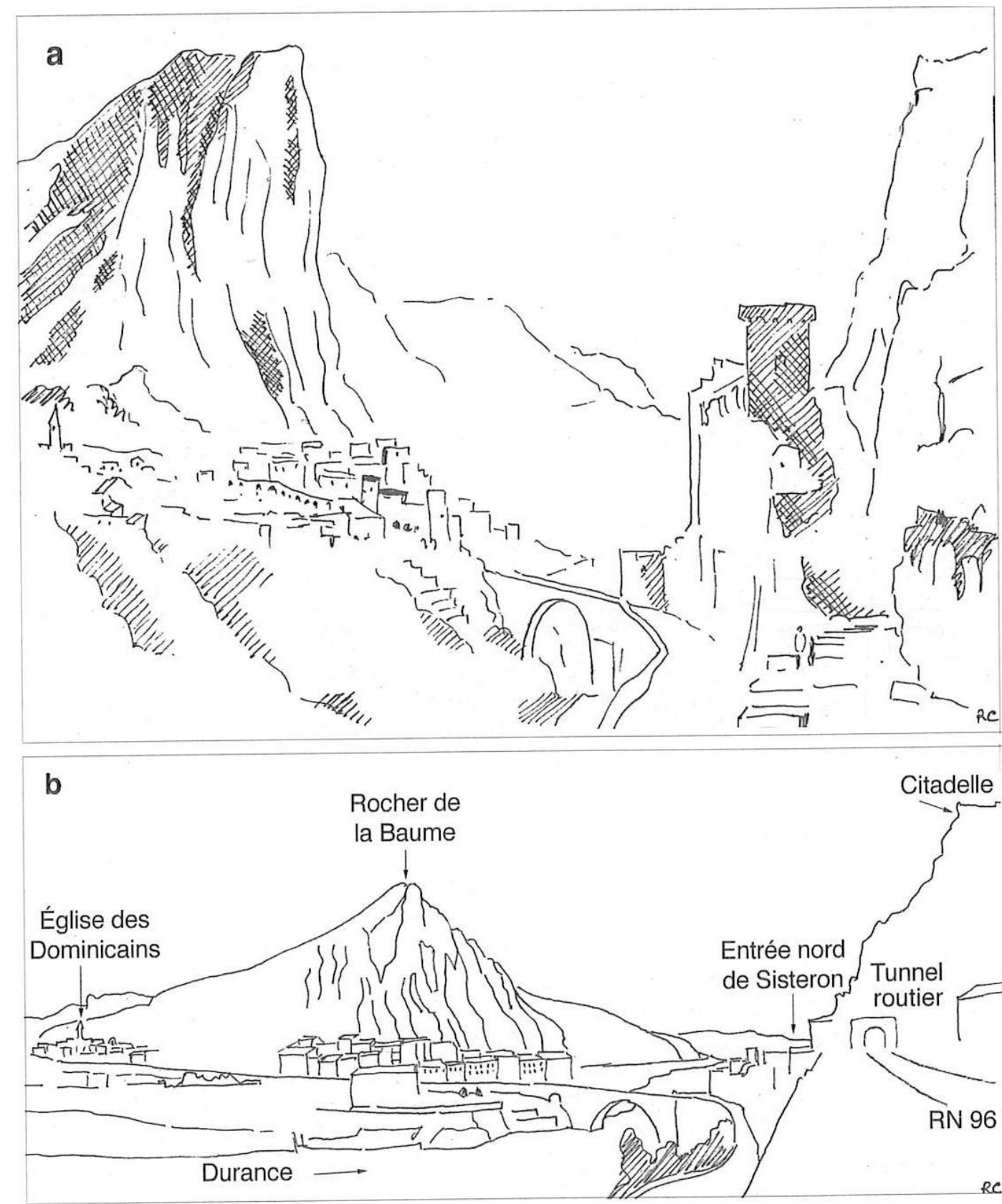

Fig.4 : La clue de Sisteron vue de l'entrée nord de la ville.

$-\mathrm{a}$ : esquisse de l'auteur d'après l'aquarelle de Turner "Une vue dans les basses Alpes: Sisteron " $(13,8$ x $19 \mathrm{~cm}$, Victoria and Albert Museum, Londres).

$-b$ : croquis d'après photo du même point de vue. 
La vue du nord, bien que peu "explorée" sur le terrain, est celle qui a donné lieu à deux magnifiques aquarelles de bonne dimension: "A view in the Basses-Alpes: Sisteron" $(13,8 \times 19 \mathrm{~cm})$ et "Sisteron" $(14 \times 18.7 \mathrm{~cm})$. Ces deux aquarelles ont été analysées par M.Guillaud (1981, p.499 à 501) mais nous revenons sur elles car elles sont caractéristiques du rôle du dessin et de l'aquarelle dans l'œuvre de Turner.

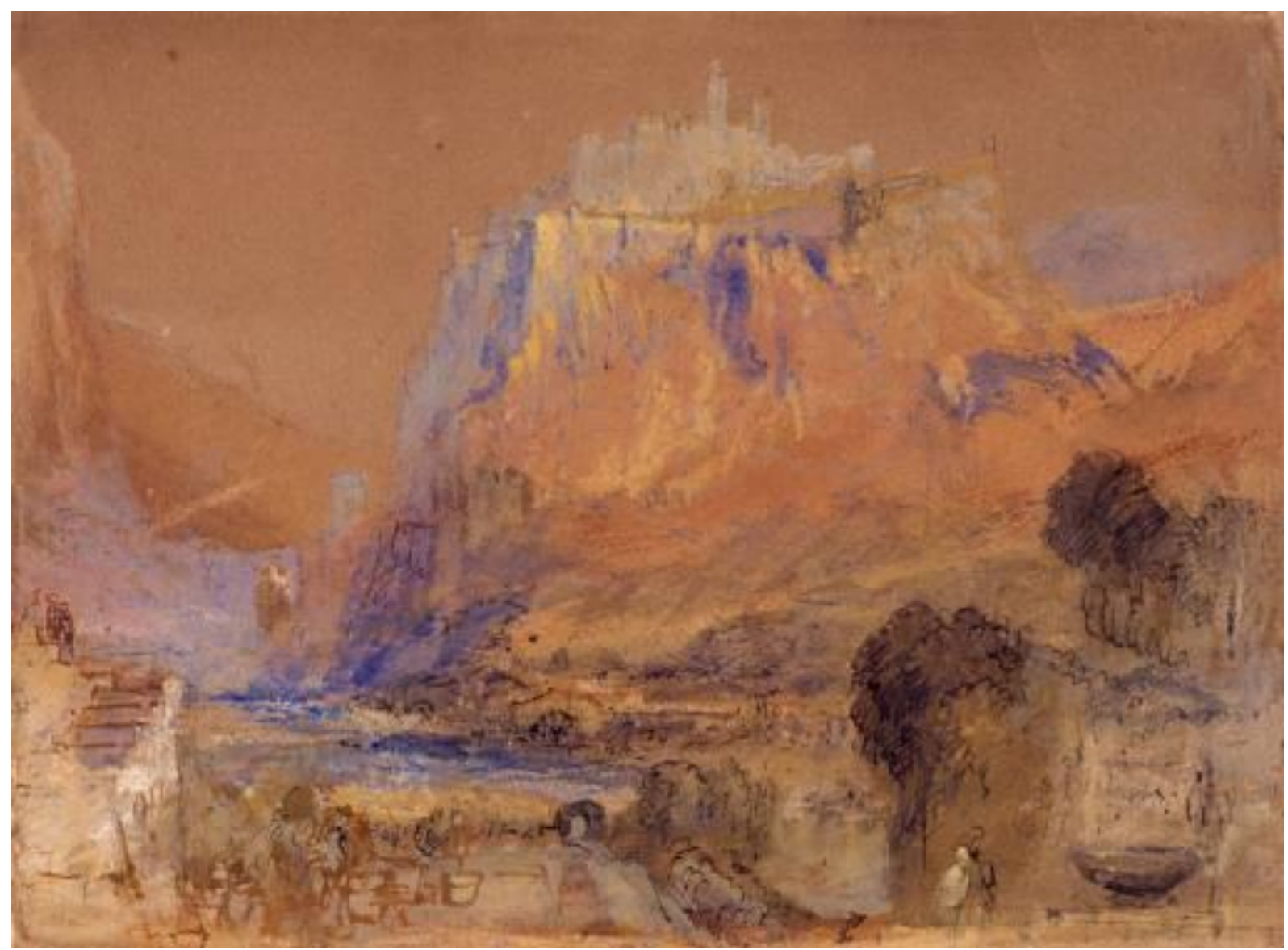

Planche IV : Sisteron, France, aquarelle avec plume sur papier chamois, 14 x 18,7cm, The Withworth Art Gallery, The University of Manchester (Crédit Photo) 

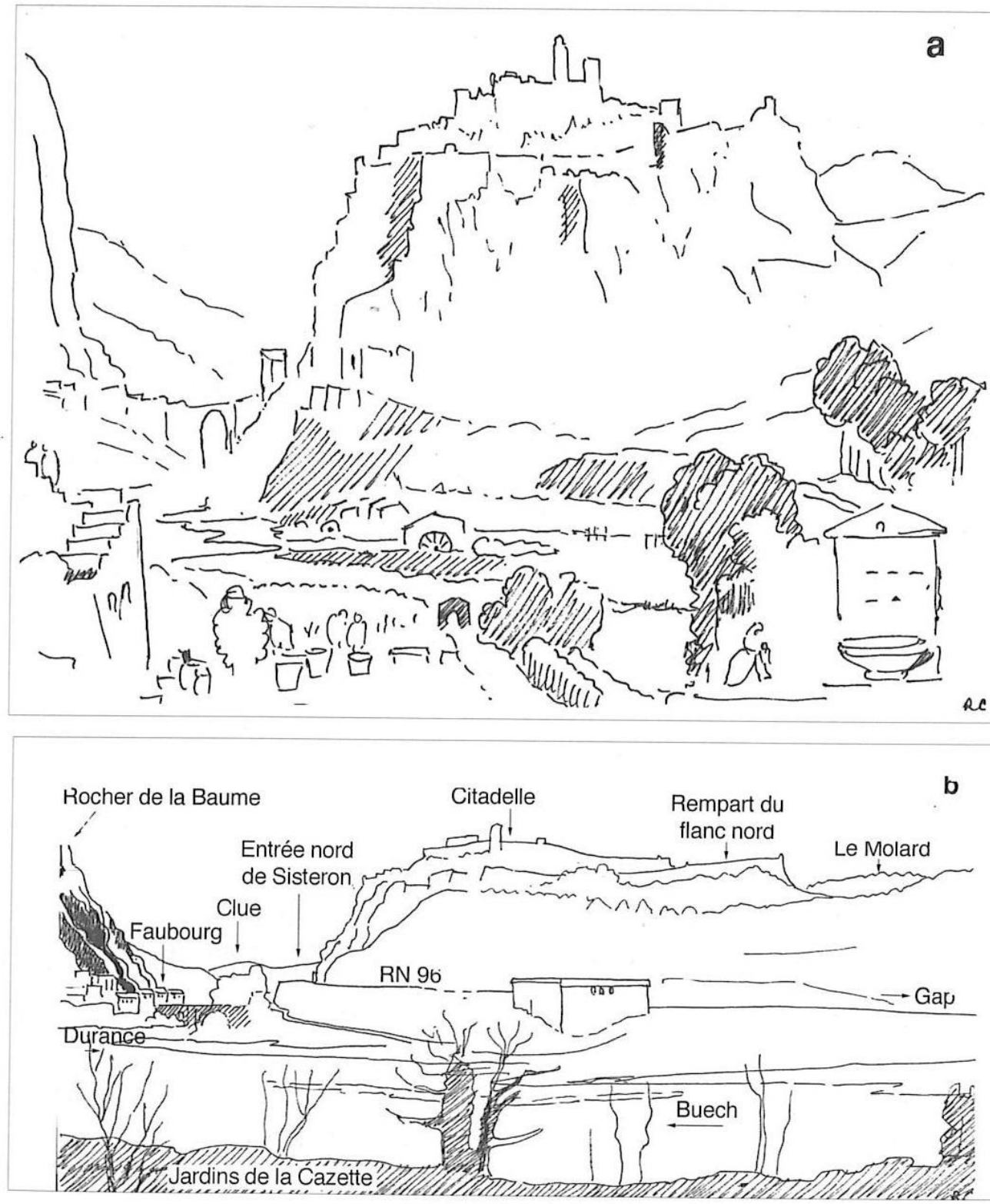

Fig.5 : Croquis schématiques de l'auteur du rocher, du château et de la clue de Sisteron, depuis les jardins de la Casette (confluence de la Durance et du Buech)

-a : d'après l'aquarelle Sisteron (planche IV)

-b : d'après photo (à comparer avec l'aquarelle de la planche IV)

La première est cadrée de très près de la route du Dauphiné, juste après la sortie de la ville (cf.fig.2, A2). L'accent est mis sur la porte fortifiée qui se dresse à la verticale au dessus du pont, et sur le rocher de la Baume, qui occupe toute la moitié gauche de l'œuvre, et s'enlève au dessus du faubourg qui fait face à la ville de l'autre côté du ravin. La comparaison de la "topographie" de l'aquarelle de Turner et de la réalité du terrain (fig. 5) montre comment l'artiste a ramassé les éléments du paysage, rapproché le clocher des Dominicains, resserré la clue, approfondi le ravin, et projeté le rocher de la Baume vers le ciel. Et cela tout en conservant des notations parfaitement réalistes : au plan du détail, les arcades de soutènement des maisons de la Baume, accrochées à la pente, sont notées; au plan de l'ensemble, le mouvement des rochers de la Baume traduisant le ploiement des couches calcaires par l'effort du plissement

La seconde aquarelle est dessinée de la même rive, mais au delà de la confluence du Buech, et c'est le rocher et la citadelle qui le surmonte qui deviennent, avec le jardin au premier plan les éléments majeurs de la composition (cf. fig.6 et 7). Sans revenir sur ce qui a déjà été largement 
étudié par les analystes de l'œuvre de Turner (D. Hill, A. Wilton, M. Guillaud, I. Warrell), regardons ce qui peut servir de leçon pour le géographe, car Turner a porté à un très haut niveau de qualité le carnet de voyage systématique : il dessinait partout, tout le temps, avec certainement une grande rapidité d'exécution, depuis la portière de sa voiture de poste sur les routes, du pont du bateau qui l'emmenait sur les fleuves, griffonnant à la hâte des profils aujourd'hui malaisés à localiser. Mais aussi il s'arrêtait pour un site particulièrement attrayant, des formes et des volumes qui pouvaient s'inscrire dans l'espace, dans la lumière, dans la couleur.

L'aquarelle se lit au moins à deux niveaux. Un niveau de détail, pas toujours aisé à observer, mais souvent d'une grande richesse, et d'une grande exactitude (du moins quant on peut la corroborer), en particulier dans les premiers plans. Ceux-ci sont animés par des scènes de vie rurale. A gauche des personnages descendent les escaliers d'une terrasse vers une sorte de clos où d'autres personnages s'activent autour de seaux et de cuveaux, devant ce qui ressemble à une vigne à l'automne (les feuilles sont jaunes, les ceps apparents), dominant la rivière (vers laquelle un petit portique de pierre permettrait de descendre): s'agit-il d'une scène de vendange ? le lieu, la terrasse fluviale dominant la rivière, et la saison rendent cette hypothèse plausible; l'année exacte de ce voyage de Turner est inconnue, mais pas la saison, car une page du carnet, utilisée antérieurement sur cet itinéraire, indique la date du 10 septembre, sur un dessin de Nice (Guillaud, 1981, p.496). A droite, séparé du clos par un muret et une haie, c'est un jardin en terrasse que nous apercevons : deux murs de soutènement, soulignés par les arbres et arbustes qui les flanquent et les couronnent, rythment la descente d'une allée vers la rivière. Une dame bien mise, avec un enfant, la remonte et s'approche d'une vasque surmontée d'un fronton de pierre décoré, adossé à la pente, comme on peut en voir dans les jardins des bastides en Provence. La place qu'il accorde à ces personnages au premier plan traduit bien le fait que " l'intérêt pour l'humanité est tel qu'il prend souvent une place majeure dans les aquarelles que Turner réalise plus tard, après le voyage(...) l'aquarelle le met en bonne place, comme élément d'un dialogue vital entre l'homme et le paysage (Guillaud, 1981, p.14).

Sur l'autre rive, plusieurs moulins à eau, dont les roues à aubes sont identifiables (au moins deux), sont accrochés à la basse terrasse de la rivière, au contact du lit majeur où alternent le bleu des souilles, le blanc des gravières et le jaune des gravons : c'est le lit "en tresses " caractéristique de la Durance et du Buech. L'un de ces moulins est attesté par une gravure du Père jésuite de Martellange, qui a dessiné la clue de Sisteron depuis l'amont, et placé un "moulin à pouldre" (poudre explosive pour les besoins de la citadelle) sur la rive droite du Buech, entre le pont de la route du Dauphiné et la confluence avec la Durance (de Gombert, 1968, p.288) ; il figure toujours sur la carte au $1 / 80.000^{\mathrm{e}}$ dressée par le service cartographique de l'armée en 1873 (coupure Digne, $\mathrm{n}^{\circ}$ 212). A l'arrière plan, la porte du Dauphiné, le pont et le faubourg de la Baume sont tout juste identifiables, à peine esquissés dans la lumière "brumeuse" du fond de la clue. Par contre le rempart du glacis nord de la citadelle, et les superstructures de la citadelle elle-même, donjon, chapelle, etc...sont bien campées, même si leurs détails échevelés exagèrent et bouleversent la stricte ordonnance de la fortification.

Il semble donc que Turner ait situé son point de vue dans le parc en terrasses, moitié domaine agricole, moitié jardin d'agrément, de la bastide de la Cazette qui se trouve accrochée à un affleurement rocheux, à la pointe du confluent Durance-Buech (cf. fig.2, A2). Même si la topographie actuelle des terrasses qui dominent le Buech ne correspond pas à celle que Turner a dessiné (les terrasses ont pu changer depuis, ou Turner a pu composer avec des éléments de croquis pris ailleurs), il est sûr que Turner, étranger de marque, a été accueilli un moment par les habitants de la bastide : il a réalisé par la suite un tableau à l'huile qui représente la maison. Il avait d'ailleurs soigneusement noté ce site sur le croquis qu'il a dessiné de la crête de la citadelle ( ${ }^{\circ} 4$, fig. 2 ).

Le second niveau d'analyse considère l'ensemble de l'œuvre : c'est celui où les formes topographiques, les volumes, les nuances et la couleur s'organisent pour faire de cette vue de Sisteron bien plus que ce qu'y verraient un historien ou un géographe. L'exagération des hauteurs 
est manifeste, et la citadelle s'enlève sur sa crête de façon vertigineuse (comme pour les coupes topographiques des étudiants de Deug). La clue devient une percée brumeuse gardée par une forteresse "romantique" dont les courtines et les tours se chevauchent dans le ciel (on pense aux lavis et aux encres des "burgs" de Victor Hugo). La mise en place des plans successifs par des nuances de teintes plus que par des contrastes de couleurs (sauf pour les ombres bleues de la pente occidentale de la clue) crée une impression d'espace entre les volumes qui favorise la circulation de la lumière.

Turner fait donc preuve de la dextérité des dessinateurs anglais du " grand tour ", celle qu'il manifeste dans ses dessins au crayon, sans éprouver d'ailleurs le besoin de reproduire partout avec la même exactitude le réel : il lui suffit de le schématiser, de le saisir par des traits vifs, qui sont les linéaments du paysage, par exemple lorsqu'il décrit les toits et les maisons dans les croquis de la ville (cf. fig. 3). Mais en même temps il y associe la force esthétique de ses recherches sur la mise en perspective des volumes entre lesquels il fait circuler la lumière, ce qui va devenir ensuite sa préoccupation majeur. Pour Maurice Guillaud, “ dans les derniers carnets le paysage n'(y) est plus qu'espace et lumière ", et dans une chronique récente du journal "Le monde " (31 octobre 2003) Philippe Dagen, à l'occasion d'une exposition de la Tate gallery à Londres sur " Turner et Venise ", parle d' "une obsession solaire contre les vues pittoresques". En ce sens, Turner, par l'abondance et la précision de ses dessins de voyage, porte l'art du dessin anglais du $18^{\mathrm{e}}$ siècle à une forme de perfection : en même temps il le dépasse en y intégrant le jeu des volumes, de l'espace et de la lumière. Il annonce ainsi les évolutions futures du paysage au $19^{\mathrm{e}}$ siècle : la vision romantique par ses mises en scène "dramatiques", et même la vision impressionniste par ses recherches sur la lumière.

\section{Conclusion : dessin d'artiste, dessin de géographe}

Il y a certainement des leçons pour le géographe dans tout cela : d'abord les carnets de voyage eux-mêmes, pour lesquels Turner se pose en précurseur. Ses seuls carnets pour la France sont au nombre de 49 et totalisent plus de 3000 dessins et croquis (Guilllaud, 1981, p.11). Chaque fois qu'il le peut, Turner tourne donc autour de son sujet, sans économiser sa fatigue : il le " mitraille" littéralement de ses croquis, comme fait le photographe aujourd'hui avec ses appareils. Ce n'est pas l'intérieur de la ville, ou les vues sur le val de Durance au nord et au sud de la clue depuis la citadelle (ce que regarde le touriste d'aujourd'hui à qui on propose l'authenticité des centres historiques, ou les points de vue les plus élevés), qui retiennent son attention, mais le site urbain, les formes et les volumes qui déboucheront pour lui sur des chefs d'œuvre picturaux (même si “ Sisteron " n'a pas dépassé le stade des aquarelles).

Ensuite le dessin, pour lequel Turner ne s'embarrasse pas de fioritures : il jette sur le papier les traits qui lui conviennent, mais qui sont toujours des choix judicieux. Le paysage se " compose" (se " recompose") sous nos yeux par un ensemble de traits légers, parfois fermes et continus, parfois jetés à la hâte, dont chacun n'a pas de signification en soi, mais dont tous concourent à la construction de l'image.

Enfin la composition des aquarelles, qui donne lieu à des exagérations de volume, des recompositions d'objets pris dans des dessins différents : cette "schématisation" du paysage est aussi une "modélisation" du paysage, comme cela avait été pressenti par Lindsay Stainton dans l'ouvrage de M. Guillaud (1981, p.300). La clue de Sisteron, si on oublie un instant le réel détaillé présent (qui n'apparaît d'ailleurs jamais au premier coup d'œil lorsqu'on les œuvres de Turner) devient sur les deux aquarelles étudiées ici un archétype de la clue.

\section{Bibliographie}

Gombert P. de, (1968) : “Napoléon de l'île d'Elbe à Sisteron ”, Ed. du SOCLE, Aix-en-Provence, 348p.

Guillaud J. et M (1981):“Turner en France”, Centre culturel du Marais, Paris, 1981, 638p. 
Hill D., (2000) “Turner, le Mont-Blanc et la Vallée d'Aoste” Région autonome de la Vallée d'Aoste, 299p.

Warrell I., (1998): “Turner, Le voyage sur la Loire ”, catalogue de l'exposition, Tate Gallery, villes de Blois et de Nantes, Réunion des musées nationaux, 256p.

Wilton A., (1979) : “J.M.W.Turner, vie et œuvre ”, Office du Livre, Fribourg, 527p.

Le corpus des œuvres étudiées :

Dessins Carnet CCXCV (Finberg) : De Gènes à Grenoble, 1835-1840, 14,4 x 9,9 cm $\mathrm{n}^{\circ} 29563$ à 29579 (classification Tate collections) 